\title{
ON A REMARKABLE FORMULA OF RAMANUJAN
}

\author{
DEBRAJ CHAKRABARTI AND GOPALA KRISHNA SRINIVASAN
}

AbSTRACT. A simple proof of Ramanujan's formula for the Fourier transform of $|\Gamma(a+i t)|^{2}$ is given where $a$ is fixed and has positive real part and $t$ is real. The result is extended to other values of $a$ by solving an inhomogeneous ODE and we use it to calculate the jump across the imaginary axis.

\section{INTRODUCTION}

The behavior of the modulus of the gamma function along vertical lines plays an important role in analytic number theory ([6, pp 767-775].) Whenever the real number $a$ is not a negative integer or zero, the function

$$
t \mapsto|\Gamma(a+i t)|^{2}
$$

is an even function in the Schwartz class, i.e., it decays rapidly at infinity along with all derivatives. When $a>0$, one can prove the following estimate due to Lerch ([3, p. 15]):

$$
|\Gamma(a+i t)|=\frac{\lambda \Gamma(1+a)}{\sqrt{a^{2}+t^{2}}} \sqrt{\frac{t}{\sinh \pi t}},
$$

where $\lambda$ satisfies $1<\lambda<\sqrt{1+t^{2}}$, and if $a=\frac{1}{2}$, we have the closed form expression

$$
\mid\left\ulcorner\left.\left(\frac{1}{2}+i t\right)\right|^{2}=\frac{\pi}{\cosh \pi t}\right. \text {. }
$$

It is natural to ask what we can say about the Fourier transform of the Schwartz class function $t \mapsto|\Gamma(a+i t)|^{2}$. The following beautiful formula was proved by Srinivasa Ramanujan in [10]. For $a>0$, we have

$$
\int_{-\infty}^{\infty}|\Gamma(a+i t)|^{2} \exp (-i \xi t) d t=\sqrt{\pi} \Gamma(a) \Gamma\left(a+\frac{1}{2}\right)\left(\cosh \frac{\xi}{2}\right)^{-2 a} .
$$

This gives the Fourier transform of the rapidly decreasing (i.e., Schwartz class) function $t \mapsto|\Gamma(a+i t)|^{2}$, and by Fourier Inversion, also the Fourier transform of $\xi \mapsto\left(\cosh \frac{\xi}{2}\right)^{-2 a}$. The existence of either of these transforms as a closed form expression is a minor miracle. Setting $a=\frac{1}{2}$ in (1.1) gives the well-known formula

$$
\int_{-\infty}^{\infty} \frac{1}{\cosh \pi t} e^{-i \xi t} d t=\frac{1}{\cosh \frac{\xi}{2}}
$$

revealing the close relation with the Gaussian function.

The integral (1.1) is the simplest in a class of integrals considered by Mellin, Ramanujan, Hardy, Hecke and others (44, p. 98, pp. 103-104, and p. 492]). Mellin

1991 Mathematics Subject Classification. 33B15.

Key words and phrases. Gamma function, Fourier transforms. 
studied integrals of this form in connection with representations of solutions of first order difference equations ([7, pp. 344-345]) and solutions of (generalized) hypergeometric equations ([8, pp 148-149]). Integrals of this form also play an important role in analytic number theory ([5, p. 152]) and in the theory of real quadratic number fields (9, p. 58]).

In this note we consider what happens to the formula (1.1) when $-1<\operatorname{Re}(a)<0$. It is clear that the formula as it stands must fail when $\operatorname{Re}(a)$ is negative and $a \notin \mathbb{Z}$ since for such $a$, the function $t \mapsto|\Gamma(a+i t)|^{2}$ is rapidly decreasing, but $\xi \mapsto \cosh ^{-2 a} \xi$ on the right hand side blows up exponentially. We obtain, using Fourier analysis, an inhomogeneous ordinary differential equation for the integral when $-1<\operatorname{Re}(a)<0$. The formula assumes a very different form when $\operatorname{Re}(a)<0$. To make the paper self-contained we offer using techniques of Fourier analysis, an alternate proof of (1.1) when $\operatorname{Re}(a)>0$.

We will prove Ramanujan's formula in the following form, where we allow $a$ to be complex:

Theorem 1.1. When $\operatorname{Re}(a)>0$,

$$
\int_{-\infty}^{\infty} \Gamma(a+i t) \Gamma(a-i t) \exp (-i \xi t) d t=\frac{2 \pi \Gamma(2 a)}{4^{a} \cosh ^{2 a}\left(\frac{\xi}{2}\right)} .
$$

When $a$ is real, the left hand side of (1.2) reduces to that of (1.1), since $\overline{\Gamma(z)}=$ $\Gamma(\bar{z})$ for all $z$ in the domain of $\Gamma$, and therefore

$$
\Gamma(a+i t) \Gamma(a-i t)=|\Gamma(a+i t)|^{2},
$$

and in the right hand side, the two expressions coincide, thanks to the classical duplication formula for the gamma function ([11, p. 35], [13, formula 3.8]):

$$
\sqrt{\pi} \Gamma(2 a)=2^{2 a-1} \Gamma(a) \Gamma\left(a+\frac{1}{2}\right) .
$$

If we try to prove Theorem 1.1 by formally computing the Fourier transform, we soon run into a familiar obstacle, namely, the appearance of an oscillatory integral

$$
\int_{-\infty}^{\infty} \exp (i \xi(x-y)) d x
$$

The standard way to cope with this (see [14, p. 37]) is to introduce a Gaussian convergence factor $\exp \left(-\epsilon x^{2}\right)$ (with $\epsilon>0$ ) in the integrand, compute the integral, and let let $\epsilon \rightarrow 0^{+}$. This is done in Section 2 below.

We then ask how to modify (1.2) when $\operatorname{Re}(a)$ is negative. We show that in Section 3 the question can be reduced to the solution of an inhomogeneous linear ODE, whose solution can be reduced to (repeated) quadratures. When $-1<$ $\operatorname{Re}(a)<0$ we prove the following:

Theorem 1.2. When $\operatorname{Re}(a) \in(-1,0)$, we have

$$
\int_{-\infty}^{\infty} \Gamma(a+i t) \Gamma(a-i t) \exp (-i \xi t) d t=
$$

$$
-\sqrt{\pi} \Gamma(a+1) \Gamma\left(a+\frac{3}{2}\right)\left\{\cosh (a \xi) \int_{0}^{\infty} \frac{e^{a s}}{\cosh ^{2 a+2}\left(\frac{s}{2}\right)} d s+\int_{0}^{\xi} \frac{\cosh (a(\xi-s))}{\cosh ^{2 a+2}\left(\frac{s}{2}\right)} d s\right\}
$$


As a consequence, we find the jump in the Fourier transform of $t \mapsto \Gamma(a-i t) \Gamma(a+$ it) as $a$ crosses the imaginary axis in Corollary 4.1

Theorem 1.2 shows that when $a<0$, the Fourier transform is no longer in closed form, but can still be expressed in terms of quadratures. It will be seen in the proof that this method can be extended to obtain similar formulas for vertical strips of the form $-(n+1)<\operatorname{Re}(a)<-n$, for any positive integer $n$ in terms of multiple quadratures.

\section{Proof of Theorem 1.1}

We begin with a useful formula that goes back to Binet ([2, p.136]). We recall that the beta function $\mathrm{B}(p, q)$ is defined for $\operatorname{Re}(p)>0$ and $\operatorname{Re}(q)>0$ to be

$$
\mathrm{B}(p, q)=\int_{0}^{1} s^{p-1}(1-s)^{q-1} d s,
$$

and the basic relation between the beta and gamma functions ([11, p. 35], [13, p. $313])$ :

$$
\Gamma(p) \Gamma(q)=\Gamma(p+q) \mathrm{B}(p, q) .
$$

Lemma 2.1 (Binet). When $\operatorname{Re}(p)>0$ and $\operatorname{Re}(q)>0$,

$$
\mathrm{B}(p, q)=\int_{-\infty}^{\infty} \frac{e^{(p-q) u}+e^{(q-p) u}}{\left(e^{u}+e^{-u}\right)^{p+q}} d u .
$$

Proof. We set $s=\left(1+e^{2 u}\right)^{-1}$ in the integral defining $\mathrm{B}(p, q)$ :

$$
\begin{aligned}
\mathrm{B}(p, q) & =2 \int_{-\infty}^{\infty} \frac{e^{2 u q}}{\left(1+e^{2 u}\right)^{p+q}} d u \\
& =\int_{-\infty}^{\infty} \frac{e^{2 u p}+e^{2 u q}}{\left(1+e^{2 u}\right)^{p+q}} d u,
\end{aligned}
$$

since the beta function is symmetric in $p$ and $q$. Multiplying the numerator and denominator of the integrand in the last integral by $\exp (-u(p+q))$, we get (2.2).

We now prove Theorem 1.1. By virtue of the beta-gamma relation (2.1):

$$
\Gamma(a+i t) \Gamma(a-i t)=\mathrm{B}(a+i t, a-i t) \Gamma(2 a) .
$$

Invoking Lemma 2.1, we see that the left hand side

$$
\int_{-\infty}^{\infty} \Gamma(a+i t) \Gamma(a-i t) \exp (-i \xi t) d t
$$

of (1.2) is equal to

$$
\begin{aligned}
& \Gamma(2 a) \int_{-\infty}^{\infty} \exp (-i t \xi)\left(\int_{-\infty}^{\infty} \frac{\left(e^{2 i t u}+e^{-2 i t u}\right)}{\left(e^{u}+e^{-u}\right)^{2 a}} d u\right) d t \\
& =\Gamma(2 a) \int_{-\infty}^{\infty} \lim _{\epsilon \rightarrow 0^{+}}\left\{\exp \left(-\epsilon t^{2}-i t \xi\right)\left(\int_{-\infty}^{\infty} \frac{\left(e^{2 i t u}+e^{-2 i t u}\right)}{\left(e^{u}+e^{-u}\right)^{2 a}} d u\right)\right\} d t,
\end{aligned}
$$

Where in the last line, we have inserted a convergence factor $\exp \left(-\epsilon t^{2}\right)$ (with $\epsilon>0$ ). Note that the function

$$
t \mapsto \int_{-\infty}^{\infty} \frac{e^{2 i t u}+e^{-2 i t u}}{\left(e^{u}+e^{-u}\right)^{2 a}} d u
$$


is rapidly decreasing, since it can be interpreted as the sum of Fourier transforms of rapidly decreasing functions, and therefore is in particular in $L^{1}$. Therefore, we are justified in applying the dominated convergence theorem, and writing (2.3) as

$$
\begin{aligned}
& \Gamma(2 a) \lim _{\epsilon \rightarrow 0} \int_{-\infty}^{\infty} \exp \left(-\epsilon t^{2}-i t \xi\right)\left(\int_{-\infty}^{\infty} \frac{e^{2 i t u}+e^{-2 i t u}}{\left(e^{u}+e^{-u}\right)^{2 a}} d u\right) d t \\
& =\Gamma(2 a) \lim _{\epsilon \rightarrow 0} \int_{-\infty}^{\infty} \frac{1}{\left(e^{u}+e^{-u}\right)^{2 a}}\left(\int_{-\infty}^{\infty}\left(e^{-i t(-2 u+\xi)-\epsilon t^{2}}+e^{-i t(2 u+\xi)-\epsilon t^{2}}\right) d t\right) d u,
\end{aligned}
$$

where in the last line the use of Fubini's theorem to interchange the order of integration is easily justified, since $t \mapsto \exp \left(-\epsilon t^{2}\right)$ is integrable. Inserting the well-known formula ([14, p. 41])

$$
\int_{-\infty}^{\infty} \exp \left(-\epsilon t^{2}-i c t\right) d t=\sqrt{\frac{\pi}{\epsilon}} \exp \left(-\frac{c^{2}}{4 \epsilon}\right)
$$

for the Fourier transform of the Gaussian into each term of the second integral factor, we get

$\sqrt{\pi} \Gamma(2 a) \lim _{\epsilon \rightarrow 0}\left\{\int_{-\infty}^{\infty} \frac{e^{\frac{-(2 u-\xi)^{2}}{4 \epsilon}}}{\sqrt{\epsilon}} \cdot \frac{1}{\left(e^{u}+e^{-u}\right)^{2 a}} d u+\int_{-\infty}^{\infty} \frac{e^{\frac{-(2 u+\xi)^{2}}{4 \epsilon}}}{\sqrt{\epsilon}} \cdot \frac{1}{\left(e^{u}+e^{-u}\right)^{2 a}} d u\right\}$

The change of variables $u=\frac{\xi}{2}+\sqrt{\epsilon} w$ transforms the first integral in (2.4) into

$$
\int_{-\infty}^{\infty} \frac{e^{-w^{2}} d w}{\left(e^{\frac{\xi}{2}+\sqrt{\epsilon} w}+e^{-\frac{\xi}{2}-\sqrt{\epsilon} w}\right)^{2 a}}
$$

Since the denominator is always greater than or equal to 1 , in this integral, the integrand is dominated by the $L^{1}$ function $w \mapsto e^{-w^{2}}$. We may therefore apply the dominated convergence theorem and conclude that the integral (2.5) converges as $\epsilon \rightarrow 0^{+}$to

$$
\sqrt{\pi} \cdot \frac{1}{\left(e^{\frac{\xi}{2}}+e^{-\frac{\xi}{2}}\right)^{2 a}}=\frac{\sqrt{\pi}}{4^{a} \cosh ^{2 a}\left(\frac{\xi}{2}\right)} .
$$

The change of variables $v=-u$ shows that the second integral in (2.4) is equal to the first for each $\epsilon>0$, and therefore, its limit as $\epsilon \rightarrow 0^{+}$is also equal (2.6), and therefore, the upshot of our computation is that the expression in (2.4), and therefore, the Fourier transform on the left hand side of (1.2) is equal to

$$
\frac{2 \pi \Gamma(2 a)}{4^{a} \cosh ^{2 a}\left(\frac{\xi}{2}\right)}
$$

which is the required right hand side.

Remark: That the first integral in (2.4) converges as $\epsilon \rightarrow 0^{+}$to the quantity in (2.6) may also be deduced from the following fact: for a fixed $\xi$, as $\epsilon \rightarrow 0^{+}$, the first factor $u \mapsto \epsilon^{-\frac{1}{2}} e^{\frac{-(2 u-\xi)^{2}}{4 \epsilon}}$ of the first integral in (2.4) converges in the sense of distributions to $\sqrt{\pi} \delta_{\frac{\xi}{2}}$, where for $p \in \mathbb{R}$, we denote by $\delta_{p}$ the delta distribution on $\mathbb{R}$ supported at the point $p$. 


\section{Proof of Theorem 1.2}

Suppose that $\operatorname{Re}(a)$ is not a negative integer or 0 . For real $\xi$, we define

$$
I(a, \xi)=\int_{-\infty}^{\infty} a \Gamma(a-i t) \Gamma(a+i t) e^{-i t \xi} d t .
$$

This is well-defined, since the integrand is easily seen to be a rapidly decreasing function of $t$. When $\operatorname{Re}(a)>0$, Theorem 1.1 along with the duplication formula (1.3) shows that

$$
I(a, \xi)=a \Phi(a) \operatorname{sech}^{2 a}\left(\frac{\xi}{2}\right)
$$

where we denote

$$
\Phi(a)=\sqrt{\pi} \Gamma(a) \Gamma\left(a+\frac{1}{2}\right),
$$

and $\operatorname{sech}(t)=(\cosh (t))^{-1}$. The basic property of the function $I(a, \xi)$ is given in the following lemma:

Lemma 3.1. If $\operatorname{Re}(a)$ is not a negative integer or zero, we have

$$
\left(\frac{d^{2}}{d \xi^{2}}-a^{2}\right) I(a, \xi)=\left(\frac{-a}{a+1}\right) I(a+1, \xi) .
$$

Proof. Using the functional relation $\Gamma(z)=\frac{\Gamma(z+1)}{z}$ twice, the right hand side of (3.1) can be rewritten as

$$
I(a, \xi)=\int_{-\infty}^{\infty}\left(\frac{a}{a^{2}+t^{2}}\right) \Gamma(1+a-i t) \Gamma(1+a+i t) e^{-i t \xi} d t .
$$

To get rid of the factor $a^{2}+t^{2}$ in the denominator in the integrand in (3.4) we differentiate under the integral twice with respect to $\xi$, and subtract $a^{2} I(a, \xi)$. We obtain

$$
\begin{aligned}
\left(\frac{d^{2}}{d \xi^{2}}-a^{2}\right) I(a, \xi)= & \int_{-\infty}^{\infty}\left(\frac{-a t^{2}}{a^{2}+t^{2}}\right) \Gamma(1+a-i t) \Gamma(1+a+i t) e^{-i t \xi} d t \\
& \quad-\int_{-\infty}^{\infty}\left(\frac{a^{3}}{a^{2}+t^{2}}\right) \Gamma(1+a-i t) \Gamma(1+a+i t) e^{-i t \xi} d t \\
& =-a \int_{-\infty}^{\infty} \Gamma(1+a-i t) \Gamma(1+a+i t) e^{-i t \xi} d t \\
& =\left(\frac{-a}{a+1}\right) \int_{-\infty}^{\infty}(a+1) \Gamma(1+a-i t) \Gamma(1+a+i t) e^{-i t \xi} d t \\
& =\left(\frac{-a}{a+1}\right) I(a+1, \xi) .
\end{aligned}
$$

We now consider the situation in which $\operatorname{Re}(a)>-1$, and $\operatorname{Re}(a) \neq 0$. Then $\operatorname{Re}(a+1)>0$, and consequently, putting the value of $I(a+1, \xi)$ from (3.2) into (3.3) we obtain that

$$
\left(\frac{d^{2}}{d \xi^{2}}-a^{2}\right) I(a, \xi)=\left(\frac{-a}{a+1}\right) \cdot(a+1) \Phi(a+1) \operatorname{sech}^{2(a+1)}\left(\frac{\xi}{2}\right) .
$$


We have thus obtained the following inhomogeneous linear differential equation satisfied by the function $\xi \mapsto I(a, \xi)$ :

$$
\left(\frac{d^{2}}{d \xi^{2}}-a^{2}\right) I(a, \xi)=-a \Phi(a+1) \operatorname{sech}^{2 a+2} \frac{\xi}{2} .
$$

The general solution of the associated homogeneous equation

$$
\left(\frac{d^{2}}{d \xi^{2}}-a^{2}\right) I(a, \xi)=0
$$

is $\xi \mapsto A e^{a \xi}+B e^{-a \xi}$, where $A$ and $B$ are constants. We proceed to find a particular solution of (3.5) by the standard method of variation of parameters. We seek the particular integral in the form

$$
\xi \mapsto v_{1}(\xi) e^{a \xi}+v_{2}(\xi) e^{-a \xi},
$$

where the functions $v_{1}$ and $v_{2}$ are determined by the system of equations

$$
\begin{aligned}
& v_{1}^{\prime}(\xi) e^{a \xi}+v_{2}^{\prime}(\xi) e^{-a \xi}=0, \\
& v_{1}^{\prime}(\xi) e^{a \xi}-v_{2}^{\prime}(\xi) e^{-a \xi}=-\Phi(a+1) \operatorname{sech}^{2 a+2} \frac{\xi}{2} .
\end{aligned}
$$

Solving the above pair of equations for $v_{1}^{\prime}$ and $v_{2}^{\prime}$, representing $v_{1}$ and $v_{2}$ as indefinite integrals, and substituting in (3.6) gives the particular integral

$$
\begin{array}{r}
-\left(\frac{\Phi(a+1)}{2} \int_{0}^{\xi} \operatorname{sech}^{2 a+2}\left(\frac{s}{2}\right) e^{-a s} d s\right) \cdot e^{a \xi}+ \\
\left(\frac{\Phi(a+1)}{2} \int_{0}^{\xi} \operatorname{sech}^{2 a+2}\left(\frac{s}{2}\right) e^{a s} d s\right) \cdot e^{-a \xi} .
\end{array}
$$

The complete solution of (3.5) is thus

$$
\begin{aligned}
I(a, \xi)= & e^{a \xi}\left(A-\frac{\Phi(a+1)}{2} \int_{0}^{\xi} \operatorname{sech}^{2 a+2}\left(\frac{s}{2}\right) e^{-a s} d s\right)+ \\
& e^{-a \xi}\left(B+\frac{\Phi(a+1)}{2} \int_{0}^{\xi} \operatorname{sech}^{2 a+2}\left(\frac{s}{2}\right) e^{a s} d s\right) .
\end{aligned}
$$

The constants $A$ and $B$ are to be determined by side conditions such as initial or boundary conditions. The Riemann Lebesgue lemma ([14, p. $106 \mathrm{ff}]$ ) which asserts that the Fourier transform of an integrable function decays at infinity, provides the requisite boundary conditions

$$
\lim _{\xi \rightarrow \infty} I(a, \xi)=0, \quad \lim _{\xi \rightarrow-\infty} I(a, \xi)=0
$$

Taking the limits as $\xi \rightarrow \infty$ and $\xi \rightarrow-\infty$ in (3.7), and using (3.8) we get the following values of $A$ and $B$, we see that if $\operatorname{Re}(a)<0$, we have

$$
A=B=-\frac{\Phi(a+1)}{2} \int_{0}^{\infty} \operatorname{sech}^{2 a+2}\left(\frac{s}{2}\right) e^{a s} d s .
$$


For use in the next section, we note that when $\operatorname{Re}(a)>0$, the same method gives us that

$$
A=B=\frac{\Phi(a+1)}{2} \int_{0}^{\infty} \operatorname{sech}^{2 a+2}\left(\frac{s}{2}\right) e^{-a s} d s .
$$

Substitution of the values of $A$ and $B$ obtained in (3.9) in (3.7), and simplifying the expression, gives us that for $-1<\operatorname{Re}(a)<0$, we have

$$
\begin{array}{r}
I(a, \xi)=-\Phi(a+1)\left\{\int_{0}^{\xi} \operatorname{sech}^{2 a+2}\left(\frac{s}{2}\right) \cosh (a(\xi-s)) d s+\right. \\
\left.\cosh a \xi \int_{0}^{\infty} \operatorname{sech}^{2 a+2}\left(\frac{s}{2}\right) e^{a s} d s\right\}
\end{array}
$$

and this is equivalent to Theorem 1.2 in view of the definitions of $I(a, \xi)$ and $\Phi(a)$.

Remark: Note that the method of proof can be used to generate formulas for $I(a, \xi)$ (and consequently the left hand side of (1.4) for $\operatorname{Re}(a)<-1$ by a recursive process. These formulas will involve repeated quadratures. For example, suppose for a positive integer $n$ that $I(a, \xi)$ has been determined for $\operatorname{Re}(a) \in(-n,-n+1)$. Then the right hand of (3.3) is known when $\operatorname{Re}(a) \in(-n-1, n)$. Consequently, we have a linear inhomogeneous ordinary differential equation to find $I(a, \xi)$ when $\operatorname{Re}(a) \in(-n-1, n)$, and this can again be solved using the method of variation of parameters, yielding a solution in terms of multiple quadratures, where the integrands now involve only elementary functions.

\section{The Jump Across the Imaginary Axis}

As we have seen in the previous section, for fixed real $\xi$, the left hand side of (1.2) is a holomorphic function of $a$, provided $\operatorname{Re}(a)$ is not a negative integer or zero, and the technique of the last section may be used to obtain formulas representing this function in terms of integrals of elementary functions. Therefore we can find the jump of this function of $a$ along the lines $\{\operatorname{Re}(a)=-n\}$, where $n$ is a non-negative integer. We now carry out this program for the simplest case of $n=0$. Denote for convenience

$$
J(a, \xi)=\int_{-\infty}^{\infty} \Gamma(a-i t) \Gamma(a+i t) e^{-i t \xi} d t,
$$

and for a point $p$ on the imaginary axis consider the jump

$$
[[J(p, \xi)]]=\lim _{\substack{a \rightarrow p \\ \operatorname{Re}(a)>0}} J(a, \xi)-\lim _{\substack{a \rightarrow p \\ \operatorname{Re}(a)<0}} J(a, \xi) .
$$

Corollary 4.1. For each real $\xi$, and for purely imaginary $p \neq 0$, we have

$$
[[J(p, \xi)]]=4 \pi \cosh (p \xi) \Gamma(2 p) .
$$

Proof. Notice that, the function $J$ above and the function $I$ of (3.1) are related by

$$
a J(a, \xi)=I(a, \xi) .
$$

Set $p=i c$, where $c \in \mathbb{R}$, and let $[[I(i c, \xi)]]$ denote the jump across the imaginary axis:

$$
[[I(i c, \xi)]]=\lim _{\substack{a \rightarrow i c \\ \operatorname{Re}(a)>0}} I(a, \xi)-\lim _{\substack{a \rightarrow i c \\ \operatorname{Re}(a)<0}} I(a, \xi) .
$$


In order to prove the result, it suffices to show that for every nonzero real $c$, we have

$$
[[I(i c, \xi)]]=2 \pi \cos c \xi \Gamma(1+2 i c) .
$$

The integrals in (3.7) are continuous functions of $a$ since the real part of $a+1$ is positive. Hence contribution to the jump of $I(\cdot, \xi)$ at the point $i c$ on the imaginary axis arises from the discontinuity in the coefficients $A$ and $B$. From equations (3.9) and(3.10), the difference in the right and left hand limits of $A e^{a \xi}$ is

$$
\begin{aligned}
e^{i c \xi} & \Phi(1+i c) 2^{2 i c+1} \int_{0}^{\infty} \frac{\left(e^{i c s}+e^{-i c s}\right) d s}{\left(e^{s / 2}+e^{-s / 2}\right)^{2 i c+2}} \\
& =e^{i c \xi} \Phi(1+i c) 2^{2 i c+1} \int_{-\infty}^{\infty} \frac{\left(e^{2 i c u}+e^{-2 i c u}\right) d u}{\left(e^{u}+e^{-u}\right)^{2 i c+2}} .
\end{aligned}
$$

Using Binet's formula with $p=2 i c+1$ and $q=1$ we simplify the last expression to

$$
2^{1+2 i c} \Phi(1+i c) e^{i c \xi} \mathrm{B}(1+2 i c, 1)=2^{2 i c} \sqrt{\pi} \Gamma(1+i c) \Gamma\left(i c+\frac{1}{2}\right) e^{i c \xi} .
$$

Using the duplication formula (1.3) to simplify the last expression, we see that the difference in the right and left hand limits of $A e^{a \xi}$ at $i c$ is

$$
\pi e^{i c \xi} \Gamma(1+2 i c) .
$$

Similarly, the difference in the right and left hand limits of $B e^{-a \xi}$ at $i c$ is

$$
\pi e^{-i c \xi} \Gamma(1+2 i c) \text {. }
$$

Thus we have

$$
[[I(i c, \xi)]]=\pi\left(e^{i c \xi}+e^{-i c \xi}\right) \Gamma(1+2 i c)=2 \pi \cos c \xi \Gamma(1+2 i c)
$$

as required.

Acknowledgment. The authors thank the referee for his helpful comments and suggestions.

\section{REFERENCES}

[1] B. C. Berndt, Ramanujan's notebooks, Part - II, Springer Verlag, New York, 1989.

[2] M. J. Binet, Mémoire sur les intégrales définies euleriennes et sur leur application à la théorie des suites, Journal de l'Ecole polytechnique, (1839) 123-343.

[3] M. Godefroy, La fonction gamma; théorie, histoire, bibliographie, Gauthier-Villars, Paris, 1901.

[4] G. H. Hardy, Collected papers - VII, Clarendon Press, Oxford, 1979.

[5] G. H. Hardy and J. E. Littlewood, Contributions to the Riemann zeta function and the theory of distribution of primes, Acta Mathematica, 41 119-196.

[6] E. Landau, Handbuch der Lehre von der Verteilung der Primzahlen - II, Chelsea publishing Co. New York, 1953.

[7] Hj. Mellin, Zur Theorie der linearen Differenzengleichungen erster Ordnung, Acta Mathematica, 15 (1891) 317-384.

[8] Hj. Mellin, Über den Zusammenhang zwischen den linearen Differential- und Differenzengleichungen, Acta Mathematica, 25 (1902) 139-164.

[9] H. Rademacher, Topics in analytic number theory, Springer Verlag, New York, 1973.

[10] S. Ramanujan, Some definite integrals, Messenger of Mathematics 44 (1915) 10-18.

[11] R. Remmert, Classical topics in complex function theory, Springer Verlag, New York, 1998.

[12] L. Schwartz, Théorie des distributions, Hermann, Paris, 1966.

[13] G. K. Srinivasan, The gamma function - an eclectic tour, American Mathematical Monthly, 114 (2007) 297-315.

[14] R. Strichartz, A guide to distribution theory and Fourier transforms, CRC press LLC, Boca Raton, 1994. 
TifR Centre for Applicable Mathematics, Sharada Nagar, Chikkabommasandra, Bengaluru- 560065, India.

E-mail address: debraj@math.tifrbng.res.in

Department of Mathematics, Indian Institute of Technology Bombay, Powai, Mumbai400076, INDIA

E-mail address: gopal@math.iitb.ac.in 